\title{
NUMERICAL SOLUTION OF TIME FRACTIONAL SCHRÖDINGER EQUATION BY USING QUADRATIC B-SPLINE FINITE ELEMENTS
}

\author{
Alahttin Esen, Orkun Tasbozan*
}

\begin{abstract}
In this article, quadratic B-spline Galerkin method has been employed to solve the time fractional order Schrödinger equation. Numerical solutions and error norms $L_{2}$ and $L_{\infty}$ are presented in tables.
\end{abstract}

\section{Introduction}

Consider the time fractional nonlinear Schrödinger equation 15]

$$
i \frac{\partial^{\gamma} U(x, t)}{\partial t^{\gamma}}+\frac{\partial^{2} U(x, t)}{\partial x^{2}}+|U(x, t)|^{2} U(x, t)=f(x, t)
$$

with the boundary conditions

$$
U(a, t)=h_{1}(t), \quad U(b, t)=h_{2}(t), \quad t \geq 0
$$

and with the initial condition

$$
U(x, 0)=g(x), \quad a \leq x \leq b,
$$

Received: 01.06.2016. Accepted: 24.08.2016. Published online: 18.12.2016.

(2010) Mathematics Subject Classification: 65D07, 65N30, 74S05, 97N40.

Key words and phrases: finite element method, Galerkin method, time fractional Schrödinger equation, quadratic B-spline.

* Corresponding author. 
where the fractional derivative is in the Caputo's sense [11, 21], $0<\gamma<1$ and $i=\sqrt{-1}$.

Equation (1.1) is solved numerically by using quadratic B-spline Galerkin method with given initial and boundary conditions. In the rest of the article we use $L 1$ approximation for $0<\gamma \leq 1$

$$
\left.\frac{\partial^{\gamma} f(t)}{\partial t^{\gamma}}\right|_{t_{m}}=\frac{(\Delta t)^{-\gamma}}{\Gamma(2-\gamma)} \sum_{k=0}^{m-1} b_{k}^{\gamma}\left[f\left(t_{m-k}\right)-f\left(t_{m-1-k}\right)\right],
$$

where $b_{k}^{\gamma}=(k+1)^{1-\gamma}-k^{1-\gamma}$.

Fractional calculus has taken part in literature in 1695 with letters which were written by two famous mathematicians G.W. Leibnitz and L' Hospital to each other. After that time, a lot of famous mathematicians such as Euler, Laplace, Fourier, Lacroix, Abel, Riemann, Liouville, Caputo have made contribution to the development of arbitrary order differentiation and integration [14]. Fractional order differentiation concept paves way for various applications and it expresses the many physical problems [19]. Recent applications(studies) show that the fractional order differential equations are very effective tools to articulate complex events and modelling many physical, engineering phenomenons [21]. There are many applications of these type equations various fields such as viscoelastic, biology, signal process, electromagnetic, chaos and fractals, traffic system, chemistry, control system, economics, finance and etc. [13].

Riemann-Liouville approximation which is frequently seen in the literature, is composed of fractional integral and fractional derivative that are mode by two famous mathematicians Riemann and Liouville. In this approximation, the initial conditions which are given with fractional order equations composed of limit values of Rieman-Liouville derivative at the initial point. This is the biggest disadvantage of this approximation. These initial points don't have any physical meaning. In 1967, M. Caputo presented a fractional order derivative which involves the limit values at the initial points of integer order derivatives with initial values that are given with fractional order equation. Due to the advantage of Caputo's definition, Caputo fractional derivative is preferred as fractional derivative operator in many fractional order differential equations [21].

In recent years, many scientists have been searching the analytical and numerical solutions of fractional order differential equations by using Laplace transform method [21, 14], power series method [21], Adomian decomposition method [23, 17], variational iteration method [16, 30, differential transform method [18, 2], homotopy perturbation method [10, 27], homotopy analysis method [1, 9], finite difference methods [26, 25], finite element method [8, 24, 7, 3, 4, 6, 5, 28, 29] and etc. 
Finite elements method first arose in 1960 and after that time, it has been used commonly in various fields of physics and engineering. Argyris, Clough and Zienkiewicz made contribution to this method [20]. With the development of computer in the last recent fifty years, it is seen that this method has a great importance in solving many problems which arise in physics and engineering [12].

$U(x, t)$ function which is given in fractional order Eq. (1.1) is a complex function. So $U(x, t)$ can be written

$$
U(x, t)=R(x, t)+i S(x, t)
$$

where $R(x, t)$ is the real part and $S(x, t)$ is the imaginary part of the function $U(x, t)$. Substituting Eq. 1.5 in problem 1.1)-(1.3), we obtain a fractional partial differential equation system

$$
\begin{aligned}
& \frac{\partial^{\gamma} S}{\partial t^{\gamma}}-\frac{\partial^{2} R}{\partial x^{2}}-\left(R^{2}+S^{2}\right) R=-f_{r}(x, t), \\
& \frac{\partial^{\gamma} R}{\partial t^{\gamma}}+\frac{\partial^{2} S}{\partial x^{2}}+\left(R^{2}+S^{2}\right) S=f_{I}(x, t),
\end{aligned}
$$

where

$$
\begin{aligned}
& f_{r}(x, t)=-\frac{2 t^{2-\gamma}}{\Gamma(3-\gamma)} \cos (2 \pi x)+\left(t^{6}-4 \pi^{2} t^{2}\right) \sin (2 \pi x), \\
& f_{I}(x, t)=\frac{2 t^{2-\gamma}}{\Gamma(3-\gamma)} \sin (2 \pi x)+\left(t^{6}-4 \pi^{2} t^{2}\right) \cos (2 \pi x)
\end{aligned}
$$

with the boundary conditions

$$
\begin{gathered}
R(0, t)=0, \quad R(1, t)=0, \quad t \geq 0, \\
S(0, t)=t^{2}, \quad S(1, t)=t^{2}, \quad t \geq 0,
\end{gathered}
$$

with the initial conditions

$$
R(x, 0)=0, \quad S(x, 0)=0, \quad 0 \leq x \leq 1 .
$$

Exact solutions of this system can be given as

$$
R(x, t)=t^{2} \sin (2 \pi x), \quad S(x, t)=t^{2} \cos (2 \pi x) .
$$




\section{Quadratic B-spline finite element Galerkin solutions}

In this part, to apply the method, we multiply the Eq. system $(1.6)$ with $W$ weighted function and integrate the system over the region, then we obtain the weighted integral function of the system

$$
\begin{aligned}
& \int_{0}^{1} W\left[\frac{\partial^{\gamma} S}{\partial t^{\gamma}}-\frac{\partial^{2} R}{\partial x^{2}}-\left(R^{2}+S^{2}\right) R\right] d x=-\int_{0}^{1} W f_{r}(x, t) d x, \\
& \int_{0}^{1} W\left[\frac{\partial^{\gamma} R}{\partial t^{\gamma}}+\frac{\partial^{2} S}{\partial x^{2}}+\left(R^{2}+S^{2}\right) S\right] d x=\int_{0}^{1} W f_{I}(x, t) d x .
\end{aligned}
$$

Applying partial integration, we get the weak form of the system over only one finite element $\left[x_{m}, x_{m+1}\right]$ as

$$
\begin{aligned}
\int_{x_{m}}^{x_{m+1}}\left(W \frac{\partial^{\gamma} S}{\partial t^{\gamma}}+\frac{\partial W}{\partial x} \frac{\partial R}{\partial x}\right. & \left.-\left(R^{2}+S^{2}\right) W R\right) d x \\
& =\left.W \frac{\partial R}{\partial x}\right|_{x_{m}} ^{x_{m+1}}-\int_{x_{m}}^{x_{m+1}} W f_{r}(x, t) d x \\
\int_{x_{m}}^{x_{m+1}} \operatorname{bigl}\left(W \frac{\partial^{\gamma} R}{\partial t^{\gamma}}-\frac{\partial W}{\partial x} \frac{\partial S}{\partial x}\right. & \left.+\left(R^{2}+S^{2}\right) W S\right) d x \\
& =-\left.W \frac{\partial S}{\partial x}\right|_{x_{m}} ^{x_{m+1}}+\int_{x_{m}}^{x_{m+1}} W f_{I}(x, t) d x
\end{aligned}
$$

Now, let us define the quadratic B-spline base functions. Partitioning the interval $[a, b]$ into $N$ finite elements of uniformly equal length by knots $x_{m}, m=$ $0,1,2, \ldots, N$ such that $a=x_{0}<x_{1} \cdots<x_{N}=b$ and $h=x_{m+1}-x_{m}$. We define the quadratic B-splines $Q_{m}(x),(m=-1(1) N)$, at the knots $x_{m}$ are defined over the interval $[a, b]$ by 22

$$
Q_{m}(x)=\frac{1}{h^{2}}\left\{\begin{array}{l}
\left(x_{m+2}-x\right)^{2}-3\left(x_{m+1}-x\right)^{2}+3\left(x_{m}-x\right)^{2}, \quad x \in\left[x_{m-1}, x_{m}\right], \\
\left(x_{m+2}-x\right)^{2}-3\left(x_{m+1}-x\right)^{2}, \quad x \in\left[x_{m}, x_{m+1}\right], \\
\left(x_{m+2}-x\right)^{2}, \quad x \in\left[x_{m+1}, x_{m+2}\right] \\
0, \text { otherwise. }
\end{array}\right.
$$


A basis for the functions defined over $[a, b]$ is formed by the set of splines $\left\{Q_{-1}(x), Q_{0}(x), \ldots, Q_{N}(x)\right\}$. Hence, a solution approximation $R_{N}(x, t)$ and $S_{N}(x, t)$ can be written in terms of the quadratic B-splines trial functions as:

$$
R_{N}(\xi, t)=\sum_{j=-1}^{N} \delta_{j}(t) Q_{j}(\xi), \quad S_{N}(\xi, t)=\sum_{j=-1}^{N} \sigma_{j}(t) Q_{j}(\xi),
$$

where $\delta_{m}(t)$ 's and $\sigma_{m}(t)$ 's are unknown, time dependent parameters which are going to be determined from the boundary and weighted residual conditions. Each quadratic B-spline covers three elements, thus each element $\left[x_{m}, x_{m+1}\right]$ is covered by three quadratic B-splines. For problem, the finite elements are identified with the interval $\left[x_{m}, x_{m+1}\right]$ and the elements knots $x_{m}, x_{m+1}$. When we use the nodal values $R_{m}, S_{m}, R_{m}^{\prime}, S_{m}^{\prime}$ which are given in terms of the parameters $\delta_{m}(t), \sigma_{m}(t)$

$$
\begin{aligned}
& R_{N}\left(x_{m}\right)=R_{m}=\delta_{m-1}+\delta_{m}, \\
& S_{N}\left(x_{m}\right)=S_{m}=\sigma_{m-1}+\sigma_{m}, \\
& R_{N}^{\prime}\left(x_{m}\right)=R_{m}^{\prime}=2\left(-\delta_{m-1}+\delta_{m}\right) / h, \\
& S_{N}^{\prime}\left(x_{m}\right)=S_{m}^{\prime}=2\left(-\sigma_{m-1}+\sigma_{m}\right) / h,
\end{aligned}
$$

the variations of $R_{N}(x, t), S_{N}(x, t)$ over the typical element $\left[x_{m}, x_{m+1}\right]$ is given by

$$
R_{N}(\xi, t)=\sum_{j=m-1}^{m+1} \delta_{j}(t) Q_{j}(\xi), \quad S_{N}(\xi, t)=\sum_{j=m-1}^{m+1} \sigma_{j}(t) Q_{j}(\xi) .
$$

If we take quadratic B-spline functions 2.3) instead of weighted functions and substituting the approximations 2.5 into equation system 2.2 , we get

$$
\sum_{j=m-1}^{m+1}\left(\int_{0}^{h} Q_{i} Q_{j} d \xi\right) \dot{\sigma}_{j}^{e}+\sum_{j=m-1}^{m+1}\left(\int_{0}^{h} Q_{i}^{\prime} Q_{j}^{\prime} d \xi\right) \delta_{j}^{e}-Z_{m} \sum_{j=m-1}^{m+1}\left(\int_{0}^{h} Q_{i} Q_{j} d \xi\right) \delta_{j}^{e}
$$

$$
-\left.\sum_{j=m-1}^{m+1}\left(Q_{i} Q_{j}^{\prime}\right)\right|_{0} ^{h} \delta_{j}^{e}=-\int_{0}^{h} Q_{i} \tilde{f}_{r}(\xi, t) d \xi, \quad i=m-1, m, m+1,
$$




$$
\sum_{j=m-1}^{m+1}\left(\int_{0}^{h} Q_{i} Q_{j} d \xi\right) \dot{\delta}_{j}^{e}-\sum_{j=m-1}^{m+1}\left(\int_{0}^{h} Q_{i}^{\prime} Q_{j}^{\prime} d \xi\right) \sigma_{j}^{e}+Z_{m} \sum_{j=m-1}^{m+1}\left(\int_{0}^{h} Q_{i} Q_{j} d \xi\right) \sigma_{j}^{e}
$$

$$
+\left.\sum_{j=m-1}^{m+1}\left(Q_{i} Q_{j}^{\prime}\right)\right|_{0} ^{h} \sigma_{j}^{e}=\int_{0}^{h} Q_{i} \tilde{f}_{I}(\xi, t) d \xi, \quad i=m-1, m, m+1,
$$

where $Z_{m}=R^{2}+S^{2}$ and $\dot{\delta}, \dot{\sigma}$ denote the $\gamma$ - order fractional derivatives dependent to $t$. When we take

$$
\begin{gathered}
A_{i j}^{e}=\int_{0}^{h} Q_{i} Q_{j} d \xi, \quad B_{i j}^{e}=\int_{0}^{h} Q_{i}^{\prime} Q_{j}^{\prime} d \xi, \quad C_{i j}^{e}=\left.Q_{i} Q_{j}^{\prime}\right|_{0} ^{h}, \\
D_{i}^{e}=-\int_{0}^{h} Q_{i} \tilde{f}_{r}(\xi, t) d \xi, \quad E_{i}^{e}=\int_{0}^{h} Q_{i} \tilde{f}_{I}(\xi, t) d \xi
\end{gathered}
$$

equation system (2.6)-(2.7) can be written in the matrix form

$$
\begin{gathered}
A^{e} \dot{\sigma}^{e}+B^{e} \delta^{e}-Z_{m} A^{e} \delta^{e}-C^{e} \delta^{e}=D^{e}, \\
A^{e} \dot{\delta}^{e}-B^{e} \dot{\sigma}^{e}+Z_{m} A^{e} \sigma^{e}+C^{e} \sigma^{e}=E^{e},
\end{gathered}
$$

where $\delta^{e}=\left(\delta_{m-1}, \delta_{m}, \delta_{m+1}\right)$ and $\sigma^{e}=\left(\sigma_{m-1}, \sigma_{m}, \sigma_{m+1}\right)$. Evaluating the above integrals for $i, j, k=m-1, m, m+1$, by using quadratic B-spline functions $A_{i j}^{e}, B_{i j}^{e}$ and $C_{i j}^{e}$, element matrices can be found as

$$
\begin{aligned}
A_{i j}^{e} & =\int_{0}^{h} Q_{i} Q_{j} d \xi=\frac{h}{30}\left[\begin{array}{ccc}
6 & 13 & 1 \\
13 & 54 & 13 \\
1 & 13 & 6
\end{array}\right], \\
B_{i j}^{e} & =\int_{0}^{h} Q_{i}^{\prime} Q_{j}^{\prime} d \xi=\frac{2}{3 h}\left[\begin{array}{ccc}
2 & -1 & -1 \\
-1 & 2 & -1 \\
-1 & -1 & 2
\end{array}\right], \\
C_{i j}^{e} & =\left.Q_{i} Q_{j}^{\prime}\right|_{0} ^{h}=\frac{2}{h}\left[\begin{array}{ccc}
1 & -1 & 0 \\
1 & -2 & 1 \\
0 & -1 & 1
\end{array}\right] .
\end{aligned}
$$


Also, $D_{i}^{e}$ and $E_{i}^{e}$ element matrixes can be evaluated as

$$
\begin{aligned}
& D_{i}^{e}=-\int_{0}^{h} Q_{i} \tilde{f}_{r}(\xi, t) d \xi=\left[\begin{array}{c}
-\int_{0}^{h} Q_{m-1} \tilde{f}_{r}(\xi, t) d \xi \\
-\int_{0}^{h} Q_{m} \tilde{f}_{r}(\xi, t) d \xi \\
-\int_{0}^{h} Q_{m+1} \tilde{f}_{r}(\xi, t) d \xi
\end{array}\right], \\
& E_{i}^{e}=\int_{0}^{h} Q_{i} \tilde{f}_{I}(\xi, t) d \xi=\left[\begin{array}{c}
\int_{0}^{h} Q_{m-1} \tilde{f}_{I}(\xi, t) d \xi \\
\int_{0}^{h} Q_{m} \tilde{f}_{I}(\xi, t) d \xi \\
\int_{0}^{h} Q_{m+1} \tilde{f}_{I}(\xi, t) d \xi
\end{array}\right] .
\end{aligned}
$$

From equation $Z_{m}=R_{N}^{2}+S_{N}^{2}$, by substituting the nodal values

$$
Z_{m}=\left(\delta_{m-1}+\delta_{m}\right)^{2}+\left(\sigma_{m-1}+\sigma_{m}\right)^{2}
$$

into equation (2.8), we obtain

$$
\begin{aligned}
& A \dot{\sigma}+B \delta-A\left(Z_{m}\right) \delta-C \delta=D, \\
& A \dot{\delta}-B \dot{\sigma}+A\left(Z_{m}\right) \sigma+C \sigma=E,
\end{aligned}
$$

where $\delta=\left(\delta_{-1}, \delta_{0}, \ldots, \delta_{N-1}, \delta_{N}\right)$ and $\sigma=\left(\sigma_{-1}, \sigma_{0}, \ldots, \sigma_{N-1}, \sigma_{N}\right)$. For $m=$ 1(1) $N-2$, the generalized rows of matrices $A, B, C$ can be written as

$$
A: \frac{h}{30}(1,26,66,26,1), \quad B: \frac{2}{3 h}(-1,-2,6,-2,-1), \quad C:(0,0,0,0,0),
$$

and that of $A\left(Z_{m}\right)$ as

$$
\frac{h}{30}\left(Z_{m 1}, 13 Z_{m 1}+13 Z_{m 2}, 6 Z_{m 1}+54 Z_{m 2}+6 Z_{m 3}, 13 Z_{m 2}+13 Z_{m 3}, Z_{m 3}\right),
$$

where

$$
\begin{aligned}
& Z_{m 1}=\left(\delta_{m-2}+\delta_{m-1}\right)^{2}+\left(\sigma_{m-2}+\sigma_{m-1}\right)^{2}, \\
& Z_{m 2}=\left(\delta_{m-1}+\delta_{m}\right)^{2}+\left(\sigma_{m-1}+\sigma_{m}\right)^{2}, \\
& Z_{m 3}=\left(\delta_{m}+\delta_{m+1}\right)^{2}+\left(\sigma_{m}+\sigma_{m+1}\right)^{2} .
\end{aligned}
$$

In system 2.9), if we write

$$
\delta=\frac{\delta^{n}+\delta^{n+1}}{2}, \quad \sigma=\frac{\sigma^{n}+\sigma^{n+1}}{2},
$$


Crank-Nicolson finite difference approximations instead of $\delta, \sigma$ and

$$
\begin{aligned}
& \dot{\delta}=\frac{d^{\gamma} \delta}{d t^{\gamma}}=\frac{(\Delta t)^{-\gamma}}{\Gamma(2-\gamma)} \sum_{k=0}^{n-1}\left[(k+1)^{1-\gamma}-k^{1-\gamma}\right]\left[\delta^{n-k}-\delta^{n-k-1}\right] \\
& \dot{\sigma}=\frac{d^{\gamma} \sigma}{d t^{\gamma}}=\frac{(\Delta t)^{-\gamma}}{\Gamma(2-\gamma)} \sum_{k=0}^{n-1}\left[(k+1)^{1-\gamma}-k^{1-\gamma}\right],\left[\sigma^{n-k}-\sigma^{n-k-1}\right]
\end{aligned}
$$

$L 1$ approximation instead of $\dot{\delta}_{m}, \dot{\sigma}_{m}$, we obtain $(2 N+4) \times(2 N+4)$ algebraic equation system as follows:

$$
\frac{A}{(\Delta t)^{\gamma} \Gamma(2-\gamma)} \sigma^{n+1}+\frac{1}{2}\left[B-A\left(Z_{m}\right)-C\right] \delta^{n+1}
$$$$
=\frac{A}{(\Delta t)^{\gamma} \Gamma(2-\gamma)} \sigma^{n}-\frac{1}{2}\left[B-A\left(Z_{m}\right)-C\right] \delta^{n}
$$

$$
-\frac{A}{(\Delta t)^{\gamma} \Gamma(2-\gamma)} \sum_{k=1}^{n}\left[(k+1)^{1-\gamma}-k^{1-\gamma}\right]\left[\sigma^{n-k}-\sigma^{n-k-1}\right]+D,
$$

$$
\frac{A}{(\Delta t)^{\gamma} \Gamma(2-\gamma)} \delta^{n+1}-\frac{1}{2}\left[B-A\left(Z_{m}\right)-C\right] \sigma^{n+1}
$$

$$
\begin{aligned}
= & \frac{A}{(\Delta t)^{\gamma} \Gamma(2-\gamma)} \delta^{n}+\frac{1}{2}\left[B-A\left(Z_{m}\right)-C\right] \sigma^{n} \\
& -\frac{A}{(\Delta t)^{\gamma} \Gamma(2-\gamma)} \sum_{k=1}^{n}\left[(k+1)^{1-\gamma}-k^{1-\gamma}\right]\left[\delta^{n-k}-\delta^{n-k-1}\right]+E .
\end{aligned}
$$

By vanishing the parameters $\delta_{-1}, \delta_{N}, \sigma_{-1}, \sigma_{N}$ with the help of boundary conditions given with problem, we handle $(2 N \times 2 N)$ square matrix algebraic system. To start the iteration, we have to know the initial parameters $\delta^{0}, \sigma^{0}$. After evaluating the initial parameters $\delta^{0}$ and $\sigma^{0}$, the numerical solutions can be obtained by using these parameters in the equation system 2.14 2.15 . We can attain numerical solutions at the intended time by writing these values in the $R_{N}$ and $S_{N}$ approximations. To enhance the approximation $R_{N}$ and $S_{N}$ solutions, we apply the iteration formulas

$$
\delta_{m}^{*}=\delta_{m}^{n}+\frac{1}{2}\left(\delta_{m}^{n+1}-\delta_{m}^{n}\right), \quad \sigma_{m}^{*}=\sigma_{m}^{n}+\frac{1}{2}\left(\sigma_{m}^{n+1}-\sigma_{m}^{n}\right)
$$

to nonlinear terms in Eq. system 2.14 2.15 for each time step. 
Initial parameters can be obtained as follows: The initial vectors

$$
\begin{aligned}
& \mathbf{d}^{0}=\left(\delta_{-1}, \delta_{0}, \delta_{1}, \ldots, \delta_{N-2}, \delta_{N-1}, \delta_{N}\right)^{T}, \\
& \tilde{\mathbf{d}}^{0}=\left(\sigma_{-1}, \sigma_{0}, \sigma_{1}, \ldots, \sigma_{N-2}, \sigma_{N-1}, \sigma_{N}\right)^{T}
\end{aligned}
$$

are determined with the aid of the initial and boundary conditions. It is necessary to have the initial numerical approximations $R_{N}(x, 0)$ and $S_{N}(x, 0)$ which satisfy the following conditions:

$$
\begin{aligned}
R_{N}(x, 0) & =R\left(x_{m}, 0\right), \quad m=0,1, \ldots, N, \\
S_{N}(x, 0) & =S\left(x_{m}, 0\right), \quad m=0,1, \ldots, N, \\
R_{N}^{\prime}\left(x_{0}, 0\right) & =R^{\prime}\left(x_{0}, 0\right), \quad S_{N}^{\prime}\left(x_{0}, 0\right)=S^{\prime}\left(x_{0}, 0\right) .
\end{aligned}
$$

Hence, using these conditions lead to a two-diagonal systems of matrix of the forms

$$
W \mathbf{d}^{0}=\mathbf{b}, \quad W \tilde{\mathbf{d}}^{0}=\tilde{\mathbf{b}}
$$

where

$$
W=\left[\begin{array}{ccccccc}
\frac{-2}{h} & \frac{2}{h} & & & & & \\
1 & 1 & & & & & \\
& 1 & 1 & & & & \\
& & & \ddots & & & \\
& & & & 1 & 1 & \\
& & & & & 1 & 1
\end{array}\right]
$$

and

$$
\begin{aligned}
& \mathbf{b}=\left(R^{\prime}\left(x_{0}, 0\right), R\left(x_{0}, 0\right), R\left(x_{1}, 0\right), \ldots, R\left(x_{N-2}, 0\right), R\left(x_{N-1}, 0\right), R\left(x_{N}, 0\right)\right)^{T}, \\
& \tilde{\mathbf{b}}=\left(S^{\prime}\left(x_{0}, 0\right), S\left(x_{0}, 0\right), S\left(x_{1}, 0\right), \ldots, S\left(x_{N-2}, 0\right), S\left(x_{N-1}, 0\right), S\left(x_{N}, 0\right)\right)^{T} .
\end{aligned}
$$




\subsection{Numerical Results}

In this section, considered problem is solved numerically by using Galerkin finite element method. Now, consider the time fractional nonlinear Schrödinger equation (1.1) with the boundary conditions

$$
U(0, t)=i t^{2}, \quad U(1, t)=i t^{2}, \quad t \geq 0
$$

and the initial condition

$$
U(x, 0)=0, \quad 0 \leq x \leq 1 .
$$

Additionally, the $f(x, t)$ is the form

$$
\begin{aligned}
f(x, t)=-\frac{2 t^{2-\gamma}}{\Gamma(3-\gamma)} \cos (2 \pi x)+\left(t^{6}-4 \pi^{2} t^{2}\right) \sin (2 \pi x) \\
+i\left(\frac{2 t^{2-\gamma}}{\Gamma(3-\gamma)} \sin (2 \pi x)+\left(t^{6}-4 \pi^{2} t^{2}\right) \cos (2 \pi x)\right)
\end{aligned}
$$

and the exact solution of this problem is given by 15

$$
U(x, t)=t^{2}(\sin (2 \pi x)+i \cos (2 \pi x)) .
$$

$L_{2}$ and $L_{\infty}$ error norms which are evaluated with the numerical solutions of real and imaginary parts are given for $\Delta t=0.005, \gamma=0.50, t=1$ and different values of $N$ in the Tables 1-2, respectively. Both of two tables show

Table 1. Error norms and numerical solutions of real part of the problem for $\gamma=0.50, \Delta t=0.005, t=1$

\begin{tabular}{|c|r|r|r|r|r|}
\hline$x$ & \multicolumn{1}{|c|}{$N=25$} & \multicolumn{1}{c|}{$N=30$} & \multicolumn{1}{c|}{$N=35$} & \multicolumn{1}{c|}{$N=40$} & \multicolumn{1}{c|}{ Exact } \\
\hline 0.0 & 0.000000 & 0.000000 & 0.000000 & 0.000000 & 0.000000 \\
0.2 & 0.942844 & 0.947022 & 0.949524 & 0.951150 & 0.951057 \\
0.4 & 0.581600 & 0.584759 & 0.586665 & 0.587916 & 0.587785 \\
0.6 & -0.585850 & -0.586937 & -0.587577 & -0.587970 & -0.587785 \\
0.8 & -0.945695 & -0.948541 & -0.950228 & -0.951298 & -0.951057 \\
1.0 & 0.000000 & 0.000000 & 0.000000 & 0.000000 & 0.000000 \\
\hline$L_{2} \times 10^{3}$ & 5.338936 & 2.598532 & 0.966275 & 0.170855 & \\
$L_{\infty} \times 10^{3}$ & 9.117853 & 4.239642 & 1.331924 & 0.562147 & \\
\hline
\end{tabular}

that the error norms decrease as the partition number $N$ increases in the numerical solutions obtained by Galerkin method. In Tables 3-4, the numerical of real and imaginary parts of the solutions and $L_{2}, L_{\infty}$ error norms are given, respectively for $\gamma=0.50, N=40, t=1$ and different values of $\Delta t$. From the tables, it is seen that the error norms decrease for the increasing values of $\Delta t$ 
Table 2. Error norms and numerical solutions of imaginary part of the problem for $\gamma=0.50, \Delta t=0.005, t=1$

\begin{tabular}{|c|r|r|r|r|r|}
\hline$x$ & \multicolumn{1}{|c|}{$N=25$} & \multicolumn{1}{c|}{$N=30$} & \multicolumn{1}{c|}{$N=35$} & \multicolumn{1}{c|}{$N=40$} & \multicolumn{1}{c|}{ Exact } \\
\hline 0.0 & 1.000000 & 1.000000 & 1.000000 & 1.000000 & 1.000000 \\
0.2 & 0.314710 & 0.311708 & 0.309935 & 0.308792 & 0.309017 \\
0.4 & -0.795690 & -0.802782 & -0.807021 & -0.809772 & -0.809017 \\
0.6 & -0.795791 & -0.802899 & -0.807144 & -0.809898 & -0.809017 \\
0.8 & 0.314552 & 0.311525 & 0.309739 & 0.308586 & 0.309017 \\
1.0 & 1.000000 & 1.000000 & 1.000000 & 1.000000 & 1.000000 \\
\hline$L_{2} \times 10^{3}$ & 8.643806 & 4.248708 & 1.583606 & 0.311933 & \\
$L_{\infty} \times 10^{3}$ & 14.545977 & 6.790519 & 2.133489 & 0.911772 & \\
\hline
\end{tabular}

Table 3. Error norms and numerical solutions of real part of the problem for $\gamma=0.50, N=40, t=1$

\begin{tabular}{|c|r|r|r|r|r|}
\hline$x$ & \multicolumn{1}{|c|}{$\Delta t=0.0125$} & $\Delta t=0.01$ & $\Delta t=0.008$ & $\Delta t=0.005$ & \multicolumn{1}{c|}{ Exact } \\
\hline 0.0 & 0.000000 & 0.000000 & 0.000000 & 0.000000 & 0.000000 \\
0.2 & 0.959018 & 0.956395 & 0.954297 & 0.951150 & 0.951057 \\
0.4 & 0.594085 & 0.592028 & 0.590383 & 0.587916 & 0.587785 \\
0.6 & -0.590194 & -0.589454 & -0.588861 & -0.587970 & -0.587785 \\
0.8 & -0.956777 & -0.954952 & -0.953491 & -0.951298 & -0.951057 \\
1.0 & 0.000000 & 0.000000 & 0.000000 & 0.000000 & 0.000000 \\
\hline$L_{2} \times 10^{3}$ & 5.305685 & 3.575290 & 2.191628 & 0.170855 & \\
$L_{\infty} \times 10^{3}$ & 10.472901 & 7.168726 & 4.524940 & 0.562147 & \\
\hline
\end{tabular}

Table 4. Error norms and numerical solutions of imaginary part of the problem for $\gamma=0.50, N=40, t=1$

\begin{tabular}{|c|r|r|r|r|r|}
\hline$x$ & \multicolumn{1}{|c|}{$\Delta t=0.0125$} & $\Delta t=0.01$ & $\Delta t=0.008$ & $\Delta t=0.005$ & \multicolumn{1}{c|}{ Exact } \\
\hline 0.0 & 1.000000 & 1.000000 & 1.000000 & 1.000000 & 1.000000 \\
0.2 & 0.303001 & 0.304932 & 0.306476 & 0.308792 & 0.309017 \\
0.4 & -0.824177 & -0.819376 & -0.815534 & -0.809772 & -0.809017 \\
0.6 & -0.824501 & -0.819635 & -0.815741 & -0.809898 & -0.809017 \\
0.8 & 0.302458 & 0.304500 & 0.306134 & 0.308586 & 0.309017 \\
1.0 & 1.000000 & 1.000000 & 1.000000 & 1.000000 & 1.000000 \\
\hline$L_{2} \times 10^{3}$ & 8.706987 & 5.860150 & 3.582478 & 0.311933 & \\
$L_{\infty} \times 10^{3}$ & 16.864538 & 11.544108 & 7.286787 & 0.911772 & \\
\hline
\end{tabular}

time step. Lastly the numerical solutions of real and imaginary parts, $L_{2}$ and $L_{\infty}$ error norms for the considered problem are given for $\Delta t=0.005, N=40$, $t=1$ and different values of $\gamma$ in Tables $5-6$. The $L_{\infty}$ error norms obtained by radial basis functions given in Ref. [15] and $L_{2}, L_{\infty}$ error norms of the numerical solutions of real and imaginary parts of the considered problem obtained by B-spline Galerkin method for $N=30, t=1$ are given in Tables 7-8 for $\gamma=0.10, \gamma=0.30$, respectively. The $L_{\infty}$ error norms of the numerical results that obtained by this method with the choice $\Delta t=0.008$ are smaller than the $L_{\infty}$ error norms that are given in Ref. [15]. In Figures 1-2, the absolute errors 
Table 5. Error norms and numerical solutions of real part of the problem for $\Delta t=0.005, N=40, t=1$

\begin{tabular}{|c|r|r|r|r|r|}
\hline$x$ & $\gamma=0.10$ & \multicolumn{1}{c|}{$\gamma=0.30$} & \multicolumn{1}{c|}{$\gamma=0.70$} & \multicolumn{1}{c|}{$\gamma=0.90$} & \multicolumn{1}{c|}{ Exact } \\
\hline 0.0 & 0.000000 & 0.000000 & 0.000000 & 0.000000 & 0.000000 \\
0.2 & 0.951334 & 0.951257 & 0.951010 & 0.950787 & 0.951057 \\
0.4 & 0.588199 & 0.588084 & 0.587685 & 0.587291 & 0.587785 \\
0.6 & -0.587683 & -0.587799 & -0.588207 & -0.588610 & -0.587785 \\
0.8 & -0.951110 & -0.951187 & -0.951445 & -0.951679 & -0.951057 \\
1.0 & 0.000000 & 0.000000 & 0.000000 & 0.000000 & 0.000000 \\
\hline$L_{2} \times 10^{3}$ & 0.240379 & 0.186763 & 0.271229 & 0.531091 & \\
$L_{\infty} \times 10^{3}$ & 0.468496 & 0.499439 & 0.665903 & 0.844600 & \\
\hline
\end{tabular}

Table 6. Error norms and numerical solutions of imaginary part of the problem for $\Delta t=0.005, N=40, t=1$

\begin{tabular}{|c|r|r|r|r|r|}
\hline$x$ & \multicolumn{1}{|c|}{$\gamma=0.10$} & \multicolumn{1}{c|}{$\gamma=0.30$} & $\gamma=0.70$ & \multicolumn{1}{c|}{$\gamma=0.90$} & \multicolumn{1}{c|}{ Exact } \\
\hline 0.0 & 1.000000 & 1.000000 & 1.000000 & 1.000000 & 1.000000 \\
0.2 & 0.308882 & 0.308856 & 0.308677 & 0.308454 & 0.309017 \\
0.4 & -0.809636 & -0.809678 & -0.809933 & -0.810222 & -0.809017 \\
0.6 & -0.809770 & -0.809814 & -0.810029 & -0.810235 & -0.809017 \\
0.8 & 0.308659 & 0.308632 & 0.308524 & 0.308445 & 0.309017 \\
1.0 & 1.000000 & 1.000000 & 1.000000 & 1.000000 & 1.000000 \\
\hline$L_{2} \times 10^{3}$ & 0.436366 & 0.499439 & 0.485480 & 0.846714 & \\
$L_{\infty} \times 10^{3}$ & 0.776350 & 0.328332 & 1.061380 & 1.314998 & \\
\hline
\end{tabular}

Table 7. Comparison of error norms of problem with the results from Ref. [15] for $N=30, \gamma=0.10, t=1$

\begin{tabular}{|c|c|c|c|c|}
\hline & \multicolumn{2}{|c|}{ Real } & \multicolumn{2}{c|}{ Imaginary } \\
\cline { 2 - 5 } & $L_{2} \times 10^{3}$ & $L_{\infty} \times 10^{3}$ & $L_{2} \times 10^{3}$ & $L_{\infty} \times 10^{3}$ \\
\hline$\Delta t=0.008$ & 0.5442 & 0.4981 & 0.8560 & 0.7526 \\
{$[15$} & - & 2.8536 & - & 2.1753 \\
\hline
\end{tabular}

Table 8. Comparison of error norms of problem with the results from Ref. [15] for $N=30, \gamma=0.30, t=1$

\begin{tabular}{|c|c|c|c|c|}
\hline \multirow{2}{*}{} & \multicolumn{2}{|c|}{ Real } & \multicolumn{2}{c|}{ Imaginary } \\
\cline { 2 - 5 } & $L_{2} \times 10^{3}$ & $L_{\infty} \times 10^{3}$ & $L_{2} \times 10^{3}$ & $L_{\infty} \times 10^{3}$ \\
\hline$\Delta t=0.008$ & 0.5254 & 0.4382 & 0.8846 & 0.6685 \\
{$[15$} & - & 2.8610 & - & 2.1771 \\
\hline
\end{tabular}

of real and imaginary parts solutions are presented for $\Delta t=0.005, N=40$, $t=1$ and different values of $\gamma$. 


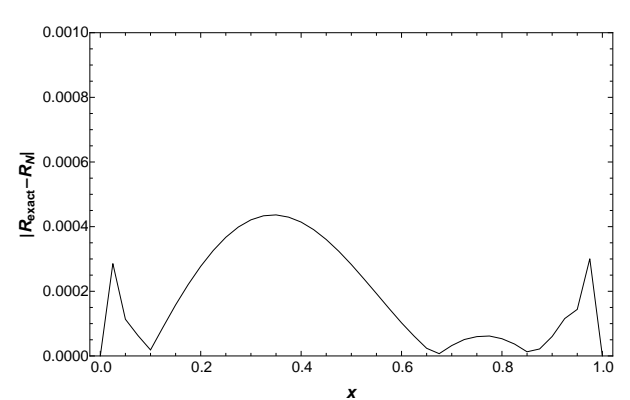

(a) $\gamma=0.10$

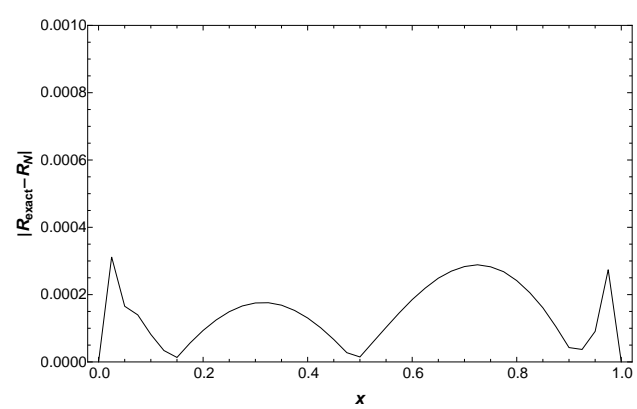

(c) $\gamma=0.50$

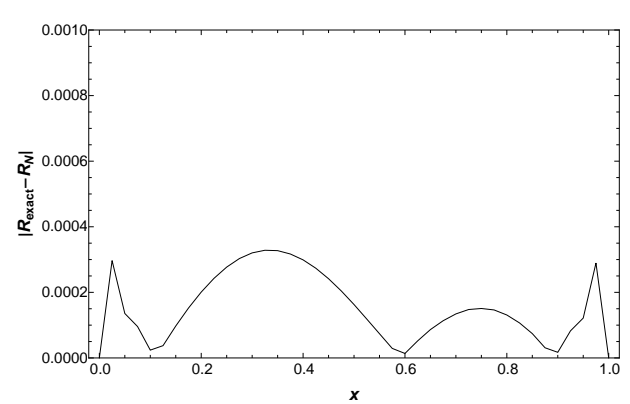

(b) $\gamma=0.30$

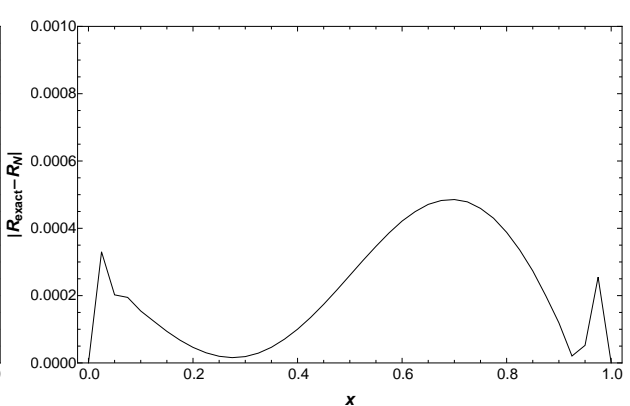

(d) $\gamma=0.70$

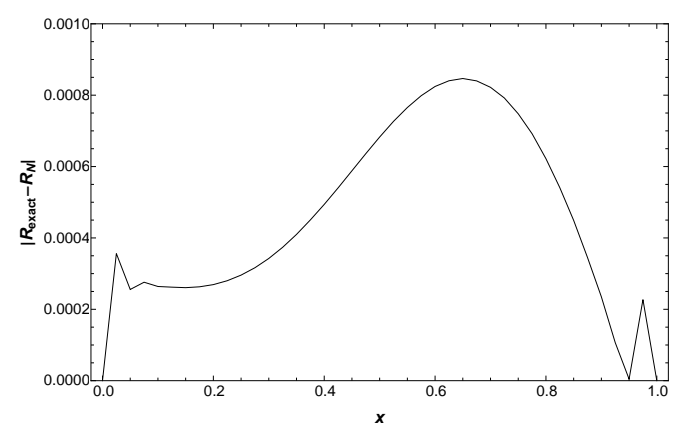

(e) $\gamma=0.90$

Figure 1. Error distributions of real part of Problem for $\Delta t=0.005, N=40, t=1$

\section{Conclusion}

In this paper, quadratic B-spline Galerkin method has been applied to achieve the numerical solutions of the time fractional Schrödinger equation. The time fractional derivative operators are made allowance for the Caputo fractional derivatives. It can be easily viewed from the numerical solutions 


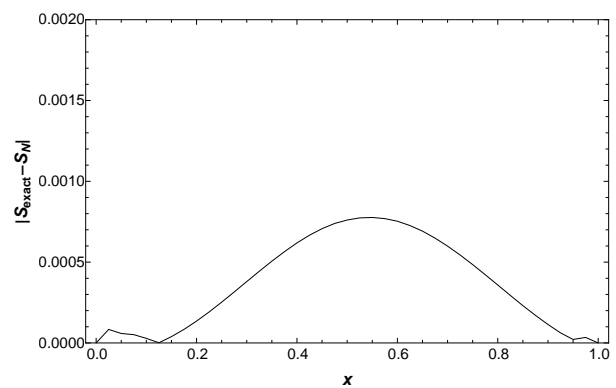

(a) $\gamma=0.10$

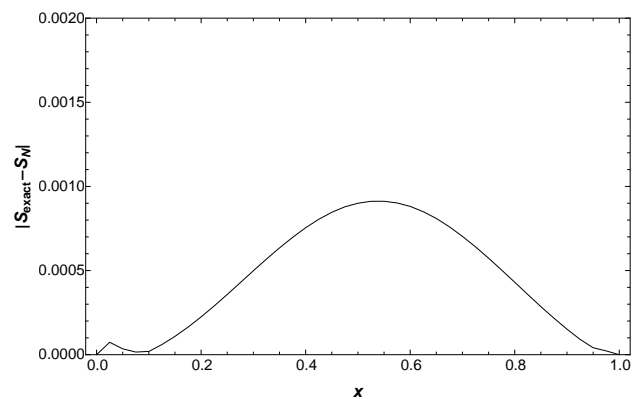

(c) $\gamma=0.50$

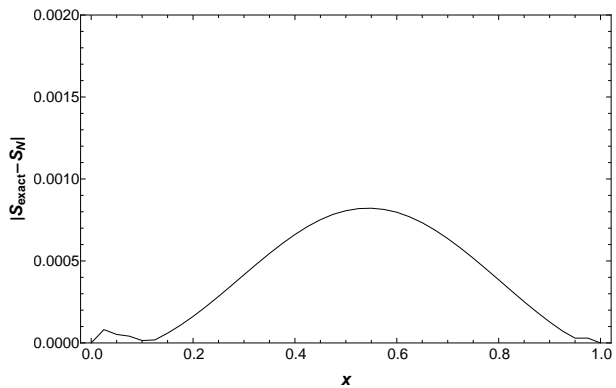

(b) $\gamma=0.30$

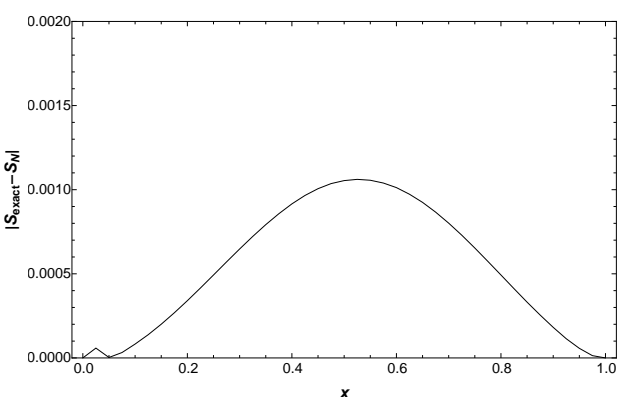

(d) $\gamma=0.70$

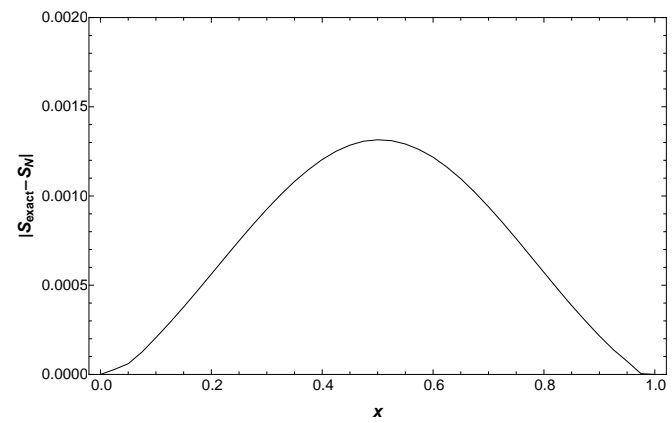

(e) $\gamma=0.90$

Figure 2. Error distributions of imaginary part of Problem for $\Delta t=0.005, N=40, t=1$

and error norms in tables obtained that this method is an extremely good to achieve numerical solutions of time fractional partial differential equations arising in physics and engineering. 


\section{References}

[1] Dehghan M., Jalil M., Abbas S., Solving nonlinear fractional partial differential equations using the homotopy analysis method, Numer. Meth. Part. D. E. 26 (2010), 448479.

[2] Ertürk V.S., Momani S., Solving systems of fractional differential equations using differential transform method, J. Comput. Appl. Math. 215 (2008), 142-151.

[3] Esen A., Tasbozan O., An approach to time fractional gas dynamics equation: Quadratic B-spline Galerkin method, Appl. Math. Comput. 261 (2015), 330-336.

[4] Esen A., Tasbozan O., Cubic B-spline collocation method for solving time fractional gas dynamics equation, Tbilisi Math. J. 8 (2015), 221-231.

[5] Esen A., Tasbozan O., Numerical solution of time fractional Burgers equation, Acta Univ. Sapientiae Math. 7 (2015), 167-185.

[6] Esen A., Tasbozan O., Numerical solution of time fractional Burgers equation by cubic B-spline finite elements, Mediterr. J. Math. 13 (20016), 1325-1337.

[7] Esen A., Tasbozan O., Ucar Y., Yagmurlu N.M., A B-spline collocation method for solving fractional diffusion and fractional diffusion-wave equations, Tbilisi Math. J. 8 (2015), 181-193.

[8] Esen A., Ucar Y., Yagmurlu N.M., Tasbozan O., A Galerkin finite element method to solve fractional diffusion and fractional diffusion-wave equations, Math. Model. Anal. 18 (2013), 260-273.

[9] Esen A., Yagmurlu N.M., Tasbozan O., Approximate analytical solution to timefractional damped Burger and Cahn-Allen equations, Appl. Math. Inf. Sci. 7 (2013), 1951-1956.

[10] Jafari H., Momani S., Solving fractional diffusion and wave equations by modified homotopy perturbation method, Phys. Lett. A 370 (2007), 388-396.

[11] Kilbas A.A., Srivastava H.M., Trujillo J.J., Theory and applications of fractional differential equations, Elsevier, Amsterdam, 2006.

[12] Logan D.L., A first course in the finite element method, Fourth edition, Thomson, Toronto 2007.

[13] Machado J.A.T., Silva M.F., Barbosa R.S., Jesus I.S., Reis C.M., Marcos M.G., Galhano A.F., Some applications of fractional calculus in engineering, Math. Probl. Eng. 2010, Article ID 639801, 34 pp., http://dx.doi.org/10.1155/2010/639801

[14] Miller K.S., Ross B., An introduction to the fractional calculus and fractional differantial equations, John Wiley, New York, 1993.

[15] Mohebbi A., Abbaszadeh M., Dehghan M., The use of a meshless technique based on collocation and radial basis functions for solving the time fractional nonlinear Schrödinger equation arising in quantum mechanics, Eng. Anal. Bound. Elem. 37 (2013), 475-485.

[16] Momani S., Odibat Z., Analytical approach to linear fractional partial differential equations arising in fluid mechanics, Phys. Lett. A 355 (2006), 271-279.

[17] Momani S., Odibat Z., Numerical comparison of methods for solving linear differential equations of fractional order, Chaos Soliton Fractals 31 (2007), 1248-1255.

[18] Odibat Z., Momani S., A generalized differential transform method for linear partial differential equations of fractional order, Appl. Math. Lett. 21 (2008), 194-199.

[19] Oldham K.B., Spainer J., The fractional calculus, Academic Press, New York, 1974.

[20] Otteson N., Pettorson H., Introduction to the finite element method, Prentice Hall, London, 1992.

[21] Podlubny I., Fractional differential equations, Academic Press, San Diego, 1999.

[22] Prenter P.M., Splines and variasyonel methods, John Wiley, New York, 1975.

[23] Shawagfeh N.T., Analytical approximate solutions for nonlinear fractional differential equations, Appl. Math. Comput. 131 (2002), 517-529. 
[24] Tasbozan O., Esen A., Yagmurlu N.M., Ucar Y., A numerical solution to fractional diffusion equation for force-free case, Abstr. Appl. Anal. 2013, Article ID 187383, 6 pp., http://dx.doi.org/10.1155/2013/187383

[25] Yuste S.B., Weighted average finite difference methods for fractional diffusion equations, J. Comput. Phys. 216 (2006), 264-274.

[26] Yuste S.B., Acedo L., An explicit finite difference method and a new von Neumanntype stability analysis for fractional diffusion equations, SIAM J. Numer. Anal. 42 (2005), 1862-1874.

[27] Wang Q., Homotopy perturbation method for fractional KdV-Burgers equation, Chaos Soliton Fractals 35 (2008), 843-850.

[28] Wei L., He Y., Zhang X., Wang S., Analysis of an implicit fully discrete local discontinuous Galerkin method for the time-fractional Schrödinger equation, Finite Elem. Anal. Des. 59 (2012), 28-34.

[29] Wei L., Zhang X., Kumar S., Yildirim A., A numerical study based on an implicit fully discrete local discontinuous Galerkin method for the time-fractional coupled Schrödinger system, Comput. Math. Appl. 64 (2012), 2603-2615.

[30] Wua G.C., Baleanu D., Variational iteration method for the Burgers flow with fractional derivatives-New Lagrange multipliers, Appl. Math. Model. 37 (2013), 6183-6190.

Department of Mathematics

INÖNÜ UNIVERSITY

Malatya, 44280

TURKEY
Department of Mathematics

Mustafa Kemal University

HATAY, 31000

Turkey

e-mail: orkun.tasbozan@inonu.edu.tr 